\title{
Mirror-enhanced super-resolution microscopy
}

\author{
Xusan Yang ${ }^{1, \star}$, Hao Xie ${ }^{1,2, \star}$, Eric Alonas ${ }^{2, \star}$, Yujia Liu ${ }^{1,3}$, Xuanze Chen ${ }^{1}$, Philip J Santangelo ${ }^{2}$, Qiushi Ren ${ }^{1}$, \\ Peng $\mathrm{Xi}^{1,2,3,4}$ and Dayong Jin ${ }^{3,4}$
}

Axial excitation confinement beyond the diffraction limit is crucial to the development of next-generation, super-resolution microscopy. STimulated Emission Depletion (STED) nanoscopy offers lateral super-resolution using a donut-beam depletion, but its axial resolution is still over $500 \mathrm{~nm}$. Total internal reflection fluorescence microscopy is widely used for single-molecule localization, but its ability to detect molecules is limited to within the evanescent field of $\sim 100 \mathrm{~nm}$ from the cell attachment surface. We find here that the axial thickness of the point spread function (PSF) during confocal excitation can be easily improved to $110 \mathrm{~nm}$ by replacing the microscopy slide with a mirror. The interference of the local electromagnetic field confined the confocal PSF to a 110-nm spot axially, which enables axial super-resolution with all laser-scanning microscopes. Axial sectioning can be obtained with wavelength modulation or by controlling the spacer between the mirror and the specimen. With no additional complexity, the mirror-assisted excitation confinement enhanced the axial resolution six-fold and the lateral resolution two-fold for STED, which together achieved 19-nm resolution to resolve the inner rim of a nuclear pore complex and to discriminate the contents of $120 \mathrm{~nm}$ viral filaments. The ability to increase the lateral resolution and decrease the thickness of an axial section using mirror-enhanced STED without increasing the laser power is of great importance for imaging biological specimens, which cannot tolerate high laser power.

Light: Science \& Applications (2016) 5, e16134; doi:10.1038/lsa.2016.134; published online 17 June 2016

Keywords: confocal; interference; point spread function; super-resolution

\section{INTRODUCTION}

The confinement of excitation light is the key for optical microscopy to resolve sub-cellular fine structures against their neighboring background $^{1-3}$. To give a few classic examples, laser scanning confocal microscopy offers higher resolution than wide-field microscopy because it uses a tightly focused excitation beam and pinhole detection to reject out-of-focus background light; stimulated emission depletion (STED) microscopy offers lateral resolution beyond the optical diffraction limit because it employs a donut-beam confined excitation to project an optically saturated virtual pinhole $e^{4,5}$, and 3D superresolution can be attained through further axial modulation ${ }^{6}$; total internal reflection fluorescence (TIRF) microscopy is suitable for single-molecule localization microscopy because it takes advantage of the evanescent field to image a superficial layer of the biological cell with an imaging depth of $\sim 100 \mathrm{~nm}$, attaining sharper features of the specimen ${ }^{2}$.

Optical interference has been used to enhance the axial resolution by generating different types of excitation modulation in wide-field fluorescence microscopy (Supplementary Fig. S1 and Supplementary Table S1 $)^{7-13}$. Bailey et al. ${ }^{7}$ proposed standing-wave excitation microscopy based on mirror reflection, but because wide-field illumination was used, the interference layer was mixed with the out-of-focus region in detection. No experimental data using mirror reflection were obtained until the $\mathrm{I}^{5} \mathrm{M}$ microscopy ${ }^{14}$, where two opposing objectives were used to interfere two coherent incident beams and the fluorescent signal. Most recently, by employing a low numerical aperture (NA) objective, a mirror, and a plan-convex lens to form Newton rings, standing-wave multiplanar excitation was demonstrated for axial imaging?.

Interference of confocal excitation light during 4Pi microscopy has been demonstrated with an axial resolution of $\sim 100 \mathrm{~nm}$ (ref. 15), but it requires the precise alignment of two diffraction-limited focal spots by two opposite microscope setups. Similarly, the isotropic focusing approach utilizes a spatial light modulator (SLM) to modulate the wave front and generate two focal spots axially, and a mirror is placed in between to form the constructive interference ${ }^{10,16,17}$. The position of the mirror is critical to generate effective interference of the two 'real' focal spots; therefore, the alignment precision of the SLM-Mirror setup is required to be $<50 \mathrm{~nm}$, whereas the $4 \mathrm{Pi}$ setup requires alignment precision $<100 \mathrm{~nm}$.

Other techniques have been explored to achieve axial superresolution. Using the STED process, an angle-STED, based on a tilted

\footnotetext{
${ }^{1}$ Department of Biomedical Engineering, College of Engineering, Peking University, No. 5 Yiheyuan Road, Beijing 100871, China; ${ }^{2}$ Wallace H Coulter Department of Biomedical Engineering, Georgia Institute of Technology and Emory University, Atlanta, GA 30332, USA; ${ }^{3}$ Advanced Cytometry Labs, ARC Centre of Excellence for Nanoscale BioPhotonics (CNBP), Macquarie University, Sydney, NSW 2109, Australia and ${ }^{4}$ Institute for Biomedical Materials and Devices (IBMD), Faculty of Science, University of Technology Sydney, Sydney, NSW 2007, Australia

*These authors contributed equally to this work.

Correspondence: P Xi, Email: xipeng@pku.edu.cn

Received 24 July 2015; revised 6 March 2016; accepted 8 March 2016
} 
mirror, has been built to generate angled super-resolution excitation (Supplementary Fig. S1j) $)^{11}$, similar to that of reflected light sheet microscopy ${ }^{18}$. Axial localization of fluorescent molecules with spectrally self-interference microscopy, by mirror reflection and a large spacer $(>10 \lambda)^{8}$ or a Langmuir-Blodgett thin film as spacer with metal-induced energy transfer has been demonstrated for axial optical nanosectioning ${ }^{12}$. Most recently, the fluorescence lifetimes have been found to correlate highly with the distance of a fluorophore from a gold mirror because the nanoplasmonics interactions and quenching/ enhancement effects are highly sensitive to the distance, which has been employed for constructing a novel type of optical nanoscopy to image the membranes of live cells with super-resolution on a gold mirror surface ${ }^{13}$.

In this work, we report a highly practical approach to immediately enable laser scanning confocal microscopy and STED nanoscopy to achieve axial super-resolution and to resolve structures, such as the nuclear pore complex, within the interior of cells (see Figure 1a and Supplementary Scheme S1). We found that when a mirror is placed behind the specimen, the interference between the excitation point spread function (PSF) and its reflection creates an axially narrowed PSF away from the mirror surface, $\sim 110 \mathrm{~nm}$ in dimension. Therefore, working in the far-field regime, this mirror-enhanced axial-narrowing super-resolution (MEANS) technique enables a more than six-fold higher axial resolution than the optical diffraction limit. Our MEANS approach is equivalent to a virtual $4 \mathrm{Pi}$ configuration, but it relaxes the alignment precision and does not require wave front modulation, as in isotropic focusing. With the axial sectioning capability and two-fold improvement of the MEANS-STED super-resolution, the inner ring structure of the nuclear pore complex, as well as the tubular structure of a viral filamentous virion, has been successfully revealed with optical microscopy for the first time. Because biological specimens and the fluorescent dye can be photodamaged by high laser power, it is of particular significance that MEANS-STED has improved both the lateral and the axial resolution without increasing the laser power.

\section{MATERIALS AND METHODS}

MEANS sample preparation

In MEANS microscopy, a piece of mirror is employed in place of the microscope slide to provide the interference. Custom-made, firstsurface mirrors were used (China Daheng Group, Beijing, China). The mirror should be a first-surface mirror, with a protective $\mathrm{SiO}_{2}$ coating, and an adjustable thickness (50, 100, 150 and $200 \mathrm{~nm}$ were tested), so that the constructive interference with a high NA objective can occur within the specimen. Due to the existence of the silica layer, cells can grow normally on the mirror surface. A coverslip can be applied to seal the specimen. As shown in Supplementary Scheme 1b, we have a custom-made mirror holder that is the same size as a microscope slide so that the mirror-backed specimen can be placed easily on any commercial confocal microscope.
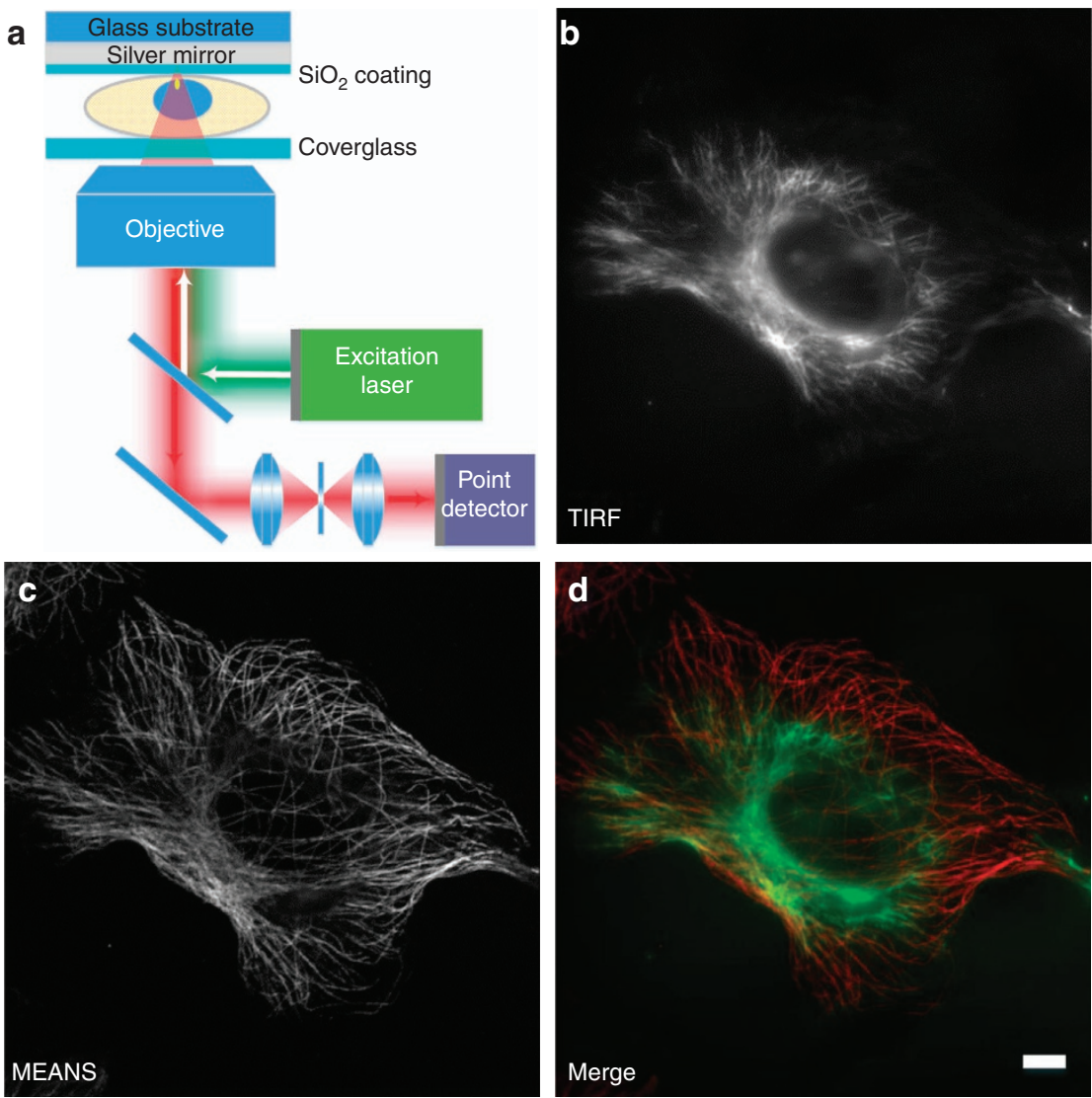

Figure 1 Schematic diagram of MEANS (a) and correlative images of a single cell acquired in TIRF (b) and MEANS (c) microscopy imaging modes. MEANS microscopy can be easily realized with a confocal microscope and takes advantage of axial interference between the incident and reflected electromagnetic field to generate an axially confined PSF $\sim 100 \mathrm{~nm}$ above the reflective mirror surface. The merged image (d) of TIRF (green) and MEANS (red) shows that the MEANS approach complements the oblique illumination-based TIRF modality by optically sectioning a cell at a different axial layer which, in this case, is close to the mirror surface. The sample here is a Vero cell immunostained for tubulin using an AlexaFluor-488 secondary antibody. Scale bar $=10 \mu \mathrm{m}$. 
Cellular sample preparation

Vero cells (CCL-81, ATCC, Manassas, VA, USA) were maintained in high-glucose DMEM (Lonza, Basel, Switzerland) with 10\% fetal bovine serum (GE Healthcare Life Sciences HyClone, Logan, UT, USA), $100 \mathrm{U} \mathrm{ml}^{-1}$ penicillin and $100 \mu \mathrm{g} \mathrm{ml}^{-1}$ streptomycin (Thermo Fisher Scientific, Waltham, MA, USA). The human respiratory syncytial virus (hRSV) strain A2 (VR-1544, ATCC, Manassas, VA, USA) was propagated in HEp-2 cells (CCL-23, ATCC) at a titer of $1 \times 10^{6}$ p.f.u. $\mathrm{ml}^{-1}$ Cells were plated the day before infection at $25 \%$ confluency. Cells were infected by removing the media, washing with phosphate-buffered saline (PBS) (without $\mathrm{Ca}^{2+}$ and $\mathrm{Mg}^{2+}$ ions, Lonza, Basel, Switzerland), adding virus at a multiplicity of infection (MOI) of 1 , and incubating the cells for $1 \mathrm{~h}$ at $37^{\circ} \mathrm{C}$. After adsorption, fresh medium was added to the inoculum. The cells were grown on top of a first-surface mirror coated with an $\mathrm{SiO}_{2}$ protective layer.

Vero cells were fixed with either $4 \%$ paraformaldehyde (VWR, Radnor, PA, USA) in PBS for $10 \mathrm{~min}$ at room temperature and then permeabilized with $0.2 \%$ Triton X-100 (for nuclear pore complex immunofluorescent staining, VWR) or with $100 \%$ ice-cold methanol for $10 \mathrm{~min}$ at $-20{ }^{\circ} \mathrm{C}$ and then permeabilized with $100 \%$ ice-cold acetone for $2 \mathrm{~min}$ at $-20^{\circ} \mathrm{C}$ (for microtubule immunofluorescent staining, VWR). Nonspecific antibody binding was blocked with 5\% bovine serum albumin (EMD Millipore, Darmstadt, Germany) in PBS for $30 \mathrm{~min}$ at $37^{\circ} \mathrm{C}$. The cells were then incubated with a primary antibody for $30 \mathrm{~min}$ at $37^{\circ} \mathrm{C}$, washed twice in PBS, incubated with a secondary antibody for $30 \mathrm{~min}$ at $37^{\circ} \mathrm{C}$, washed twice in PBS and mounted in a mixture of Mowiol 4-88 (Sigma Aldrich, St Louis, MO, USA) and DABCO (VWR $)^{19}$.

The primary antibodies used were rabbit anti-alpha tubulin (polyclonal IgG, catalog: ab18251, Abcam, Cambridge, MA, USA) and mouse anti-nuclear pore complex (NPC) proteins that contain FXFG repeats (monoclonal IgG, catalog: ab24609, Abcam). The secondary antibodies used were goat anti-rabbit DyLight 650 (Thermo Fisher Scientific, Waltham, MA, USA) and donkey anti-mouse AlexaFluor 488 (Thermo Fisher Scientific).

\section{Spin capture of RSV filaments on glass}

To capture single hRSV filamentous virions on glass, hRSV A2 was propagated in HEp-2 cells at an MOI of 0.1. At 4 days post infection, the cell-associated and supernatant fractions were scraped, freezethawed and spun through 5 and $0.45 \mu \mathrm{m}$ pore-size centrifugal filters (EMD Millipore) at $5000 \times \mathrm{g}$ and $4{ }^{\circ} \mathrm{C}$ for 4 and $1 \mathrm{~min}$, respectively. The fraction between 0.45 and $5 \mu \mathrm{m}$ in diameter was collected and immobilized onto a poly-L-lysine (Sigma Aldrich)-coated, first-surface mirror or cover glass by adsorption of $500 \mu \mathrm{l}$ of filtered virus for $2 \mathrm{~h}$ at $4{ }^{\circ} \mathrm{C}$. The immobilized virions were fixed using $4 \%$ paraformaldehyde and were immunofluorescently stained according to the aforementioned protocol. The antibodies used were anti-RSV F monocolonal (palivizumab, MedImmune, Gaithersburg, MD, USA) and anti-RSV N monoclonal (monoclonal IgG1, ab22501, Abcam). Coverslips were mounted in a mixture of Mowiol and DABCO (VWR) ${ }^{19}$.

\section{MEANS-STED}

MEANS-STED imaging was performed with a Leica TCS SP8 STED $3 \mathrm{X}$ system equipped with a white light laser as excitation and 592 and $660 \mathrm{~nm}$ for STED depletion. The HyD detector and $100 \times$ oilimmersion objective (NA 1.4) were employed. Time-gated detection was also used ${ }^{20}$, in which the AlexaFluor-488 detection is delayed $0.5 \mathrm{~ns}$, whereas the Cy5 detection is delayed $1 \mathrm{~ns}$.

\section{RESULTS AND DISCUSSION}

MEANS and TIRF microscopy reveal complementary structural information

Supplementary Fig. S1 further illustrates the schematic difference comparing the MEANS technique with a variety of available axialenhanced microscopy techniques. MEANS, similar to TIRF by providing wide-field microscopy with axial confinement and improved contrast, can confine the axial PSF depth of confocal microscopy to $\sim 100 \mathrm{~nm}$, with doubled signal intensity. Furthermore, MEANS complements the TIRF imaging modality by optically sectioning two different super-resolution layers: TIRF is responsible for imaging the sample layer close to the coverslip, whereas MEANS optically sections the layer that is close to the mirror (interior of the specimen). In Figure 1b-1d, we demonstrate a series of in situ correlative images of TIRF and MEANS from a single Vero cell. Whereas the layer near the coverslip can be imaged by TIRF (or HiLo mode ${ }^{21}$ because the refractive index of the medium is larger than the NA of the objective, Figure 1b), the MEANS imaging modality (Figure 1c) provides superresolution information within the interior layer of the cells with a $\sim 110 \mathrm{~nm}$ axial thickness but a distance of $\sim 100 \mathrm{~nm}$ from the mirror surface (wavelength dependent), making MEANS more suitable for the study of problems that are not at the cell membrane but localized more deeply within the cell. When the MEANS image is overlaid with a complementary TIRF image (Figure 1d), the spatial relationship between the two images is clear (see also wide-field in Supplementary Fig. S2, in which both layers can be visualized simultaneously).

\section{Theoretical simulation of the MEANS and MEANS-STED PSFs}

To predict the axial confinement of the PSF, we simulated the excitation electromagnetic field for multiple imaging techniques (Figure 2). Our simulation results of the electromagnetic field of the MEANS microscopy revealed that, the local maximum intensity for MEANS (Figure 2c) is approximately four times that of conventional confocal microscopy (Figure 2a) and approximately two times that of $4 \mathrm{Pi}$ (Figure $2 \mathrm{~b}$ ). Because the local maximum is a result of constructive interference, MEANS can generate local EM-field enhancement over a long focal distance (Supplementary Video S1), relieving the need for precise alignment of the mirror.

\section{Demonstration of optical sectioning using MEANS and excitation scanning}

Because the distance between the mirror and the MEANS-enhanced layer is linearly proportional to the excitation wavelength, we can realize optical sectioning within the specimen with MEANS through excitation wavelength modulation (MEANS-excitation scanning optical nanosectioning, MEANS-ESON), which has been experimentally verified using MEANS to axially section fluorescent nanodiamonds by scanning the excitation wavelength and taking advantage of the broad absorption spectrum of the nitrogen vacancy centers within nanodiamonds. A laser scanning confocal system (Leica TCS SP8) equipped with a white light laser and a plan-apochromatic objective (Leica HC PL APO CS2, 63x/1.40 OIL) has been used. As shown in Figure 3, three scanning results using different excitation bands resulted in different layers of nanodiamond nanoparticles (pseudocolor is used to indicate different layers of nanoparticles scanned by different excitation color). If the nanodiamonds are in the same layer, then the color should be identical. However, from the arrows, we can see that some of the nanodiamonds are yellow as the dominant color (which is a combination of $\mathrm{R}$ and $\mathrm{G}$ ), whereas others are strongly blue (which locates mainly in the B layer, closer to the mirror). 

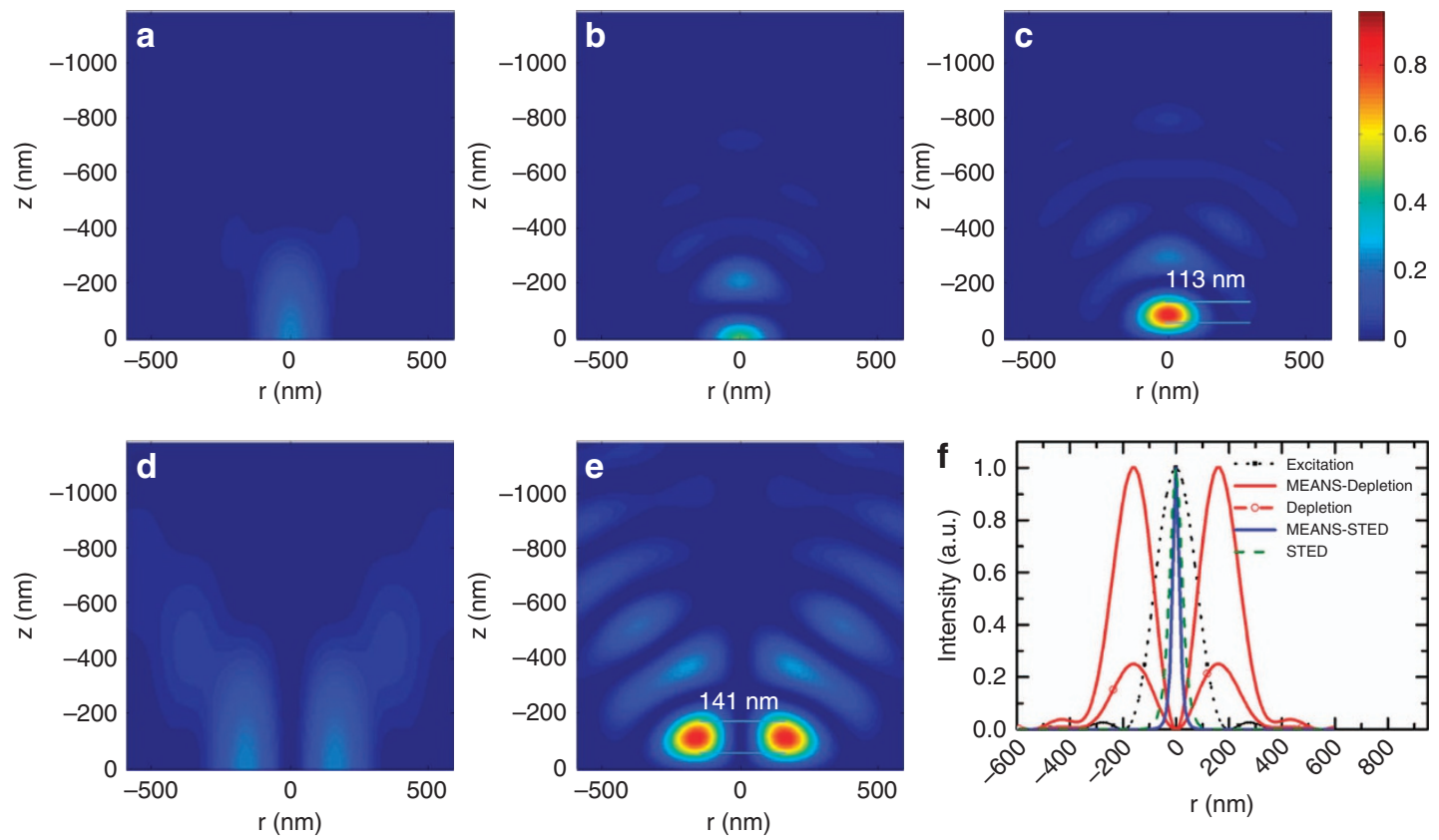

Figure 2 Theoretical simulation results of the focal intensity profiles of: (a) confocal excitation, (b) 4Pi excitation, (c) MEANS excitation, (d) STED depletion and (e) MEANS-STED depletion. The origin of the z-axis denotes the center of PSFs in a-c and the mirror position in $\mathbf{d}$ and $\mathbf{e}$. Whereas confocal microscopy can generate a PSF with $700 \mathrm{~nm}$ axial thickness, 4Pi and MEANS can generate PSFs with $\sim 110 \mathrm{~nm}$ axial thickness, benefitting from axial interference. Objectives with $n=1.5, N A=1.4$, and $\lambda_{\text {ex }}=488 \mathrm{~nm}$ and $\lambda_{\text {dep }}=592 \mathrm{~nm}$ are used for simulation. The local maximum intensity for MEANS $\mathrm{d}$ is approximately four times that of conventional confocal microscopy a and two times that of $4 \mathrm{Pi} \mathbf{b}$ because in $4 \mathrm{Pi}$, the beam is split into two and then recombined, whereas MEANS takes full advantage of the incident intensity through reflection. Because the intensity of depletion is improved by 3.6-fold, close to two-fold resolution enhancement over conventional STED can be obtained for MEANS-STED, as shown in (f).

Furthermore, taking advantage of the virtual PSF, MEANS modality has only one sidelobe with which to generate images with sharper contrast, whereas the PSF of 4Pi has two symmetric sidelobes ${ }^{22}$, which requires deconvolution for data representation. In our MEANS approach, after passing through the confocal pinhole, the only sidelobe is largely suppressed, making the image very sharp.

\section{Verification of the axial confinement thickness}

To verify the improved axial PSF confinement of $110 \mathrm{~nm}$, we prepared a phantom specimen by embedding $20 \mathrm{~nm}$ fluorescent beads inside low-concentration agarose, which was then evaluated by MEANS confocal microscopy. As shown in Supplementary Fig. S3, a three-fold enhancement in the signal-to-background ratio (SBR) was achieved at the 13-14- $\mu \mathrm{m}$ layer primarily because with the decrease of the PSF, the fluorescent signal increases proportionally due to the higher excitation energy confinement. We have also simulated the effect of the integrated energy enhancement (detection photon number) for MEANS with different confocal pinhole sizes, and the simulation further validated our experimental results.

\section{Dual-color MEANS microscopy}

To further verify the above simulation results and to demonstrate the robustness of the MEANS modality, we applied the MEANS approach on a commercial laser scanning confocal microscope (single- and twophoton excitation) as well as a spinning-disk microscope. We acquired a series of images along the $\mathrm{z}$-direction of the microtubules of a Vero cell grown on the mirror substrate. The images for confocal, twophoton and spinning disk approaches are shown in Supplementary Figs S4-S6, respectively. The dual-color imaging of microtubules and NPCs in a Vero cell is shown in Figure 4. In these figures, image stacks are acquired by moving the objective toward the specimen.
According to our simulation result (Supplementary Video S1), the position of the MEANS confinement focal spot is fixed $\sim 100 \mathrm{~nm}$ above the mirror surface during mirror translation. The layers from 2$2.5 \mu \mathrm{m}$ in Figure 4 and Supplementary Fig. S4 belong to the MEANS modality region and show almost identical images due to the fixed MEANS focal spot. The optical sectioning layers above this $0.5 \mu \mathrm{m}$ region belong to the conventional confocal microscopy modality. These quantitative imaging results indicate that the MEANS-confocal modality has a tolerant region of $\sim 0.4 \mu \mathrm{m}$ to section an axially confined thin layer of the cell, $100 \mathrm{~nm}$ from the mirror, which is consistent with our simulation result (Supplementary Video S1). Comparing the MEANS image (Figure $4 \mathrm{f}$ ) to the confocal images (Figure $4 \mathrm{a}-4 \mathrm{c}$ ), the MEANS with excitation intensities enhanced by interference can significantly enhance the image SNR through its increased fluorescence signal intensity, which suggests that the conventional axial resolution measurement, in which the specimen translates relative to the objective will not work in MEANS because the PSF does not move with the objective.

\section{Moving the MEANS layer within the cell}

To show the possibility of imaging at different depths into a cell, we grew Vero cells on mirrors coated with different thicknesses of silica. Figure 5 shows different optical sectioning layers of actin filaments within the Vero cells, achieved by changing the thickness of the silica coating from 50, 100, 150 and $200 \mathrm{~nm}$. Actin microfilaments, one type of cytoskeletal element, are responsible for cell shape and cell motility. Figure $5 \mathrm{c}$ and $5 \mathrm{~d}$ shows the optical sections of the MEANS layer as it is moved closer and closer to the cell surface membrane when the thickness of silica spacer is increased from 50 to $150 \mathrm{~nm}$. By rigorously examining the $50 \mathrm{~nm}$ layers, we found that all images (typically shown in Figure 5b) were rich in actin filaments and bundles but with very 
a
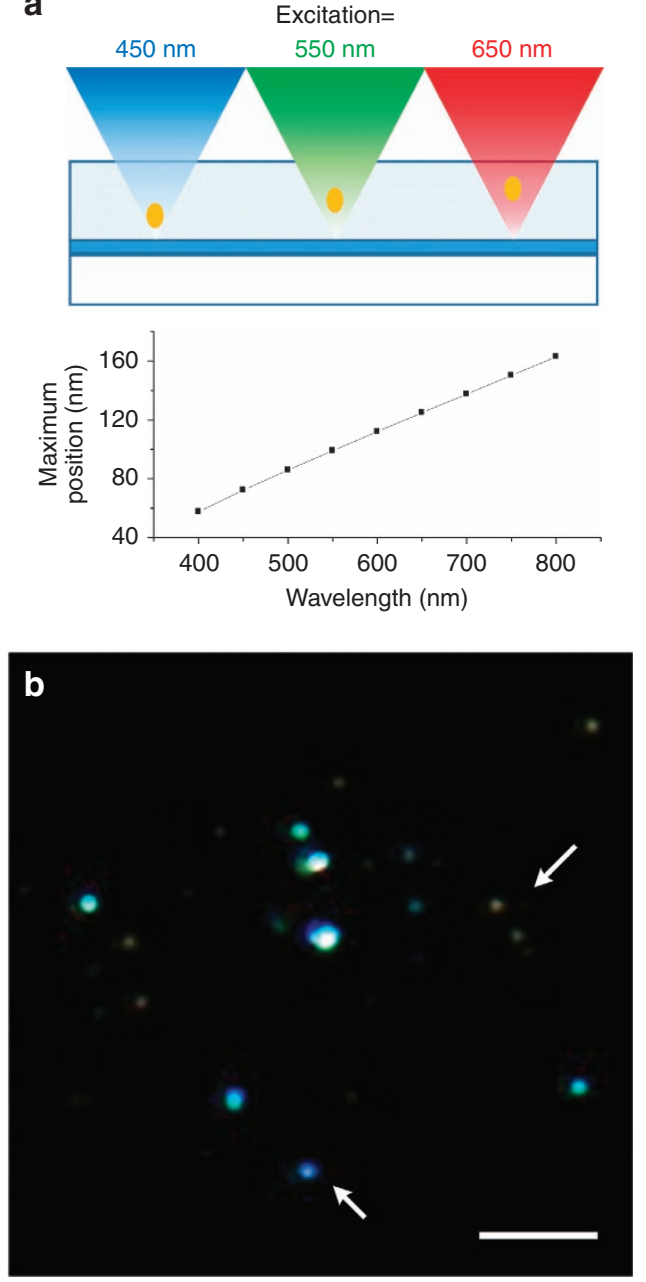

Figure $\mathbf{3}$ Imaging of nanodiamond particles embedded in agarose with different excitation wavelengths. Excitation with longer wavelength results in a further PSF relative to the mirror. In the lower RGB image, the fluorescence excited by $470-490,550-570$ and $650-670 \mathrm{~nm}$ is mapped to the $B, G$ and $R$ channels, respectively. Arrows show beads with different colors, which indicates that they are at different depths and are differentiated by MEANS-excitation scan optical nanosectioning (MEANSESON). Scale bar $=5 \mu \mathrm{m}$.

little blank shadow of the nuclei. The images from the 100 and $150 \mathrm{~nm}$ layers (Figure $5 \mathrm{c}$ and $5 \mathrm{~d}$ ) show clear elliptical shadows of the nuclei free of the actin filaments inside. In addition, the margins of the cell are more apparent in Figure $5 \mathrm{c}$ and $5 \mathrm{~d}$ compared with the $50 \mathrm{~nm}$ in Figure 5b. After further increasing the thickness of the silica spacer to $200 \mathrm{~nm}$, the images contain both actin and nuclei (Figure 5e), similar to the control images taken by conventional confocal microscopy (Figure $5 f$ ), suggesting that the axial super-resolution power from the MEANS modality disappears when the silica spacer is more than $200 \mathrm{~nm}$. In addition, MEANS is tolerant to small-angle misalignments of the mirror relative to the objective (Supplementary Fig. S7).

\section{MEANS-STED microscopy of the NPC and viral filaments}

Another significant advantage of the MEANS modality is its compatibility with STED microscopy. According to our simulation results (Figure $2 \mathrm{~d}$ and $2 \mathrm{e}$, and Supplementary Video S2), the local electromagnetic field for the vortex-modulated 'doughnut' STED PSF is axially confined to form a 163-nm MEANS-STED depletion PSF. Therefore, both the intensity of the donut-shaped depletion beam and the excitation beam ${ }^{23-25}$ have been enhanced by 3.6-fold within the MEANS region. Because the resolution of STED is dependent on the depletion intensity, which can be written as ${ }^{26,27}$

$$
d=\frac{\lambda}{2 \mathrm{NA}} \frac{1}{\sqrt{1+I_{\mathrm{dep}} / I_{\mathrm{sat}}}}
$$

where $I_{\mathrm{dep}}$ is the depletion intensity and $I_{\text {sat }}$ is the saturation intensity of the molecule, the constructive interference in MEANS-STED mode provides close to two-fold resolution enhancement over conventional STED.

To verify our MEANS method in STED nanoscopy, we applied the MEANS approach in a commercial Leica TCS SP8 STED 3X superresolution microscope. As a result of the MEANS-assisted STED (Figure 6a-6d), we achieved 19-nm resolution to visualize the inner rim of the NPC using a relatively low 592-nm depletion laser power of $60 \mathrm{~mW}$ measured at the back aperture of the objective. The NPC restricts the diffusion of molecules between the nucleoplasm to the cytoplasm and is composed of three concentric ring nucleoporins (Nups) held together by linker proteins. The inner channel is composed of the phenylalanine-glycine (FG) Nups ${ }^{28}$. Because of the small diameter of FG Nups $(\sim 50 \mathrm{~nm})$, its ring structure has only been revealed by electron microscopy from isolated NPC complexes ${ }^{29,30}$. Even when other super-resolution techniques, such as dSTORM, were utilized with localization precision of $20 \mathrm{~nm}$, the ring structure was unresolvable $^{31}$. After the inner channels (including Nup62, Nup95 and Nup110 proteins) of the NPCs were stained, MEANS in conjunction with a commercial STED system enabled us to record one of the first optical images of an FG Nup in a mammalian cell (Figure 6a-6d). The measured diameter relative to the size of the whole nuclear pore complex confirmed that the antibody was indeed bound to Nups in the center of the $\mathrm{NPC}^{29}$. The resolution of $19 \mathrm{~nm}$ is one of the new record of STED super-resolution in biological applications ${ }^{32-36}$. The resolution improvement is largely because two-fold resolution enhancement can be obtained without increasing the depletion laser power. Because biological specimens and fluorescent dyes are generally very sensitive to high power laser, this is a very important advance in order to dramatically improve the resolution without increasing the laser power. A comparison with conventional 3D STED can be found in Supplementary Fig. S8.

The axial super-resolution achieved by the MEANS approach has further assisted STED for 3D super-resolution visualization of the submicron hollow structures. hRSV produces filamentous virions that are $100-200 \mathrm{~nm}$ in diameter and up to several microns in length. The distance between the centroid of the fusion $(\mathrm{F})$ proteins was measured as $\sim 120 \mathrm{~nm}$ by dSTORM ${ }^{37}$. To use this model system to test MEANSSTED, we immunofluorescently stained the F protein, which is in the virion membrane, and the nucleoprotein $(\mathrm{N})$, which is bound to the genomic RNA inside the virion space. Without the mirror, despite using even higher STED depletion power $(108 \mathrm{~mW}$ at $592 \mathrm{~nm})$ than the MEANS-STED $(72 \mathrm{~mW})$, the conventional STED with the axial PSF of over $500 \mathrm{~nm}$ cannot resolve the F protein on the two opposing sides of the membrane (Figure $6 \mathrm{f}-6 \mathrm{i}$ ) due to contaminating light from the F protein present on the top and bottom of the filament. With the MEANS implementation (Figure 61-6o), a thin section in the middle of the filament is excited, and the top and bottom of the filament are not excited, allowing us to resolve the F proteins on opposing sides of the viral envelope with the $\mathrm{N}$ protein in the center. 

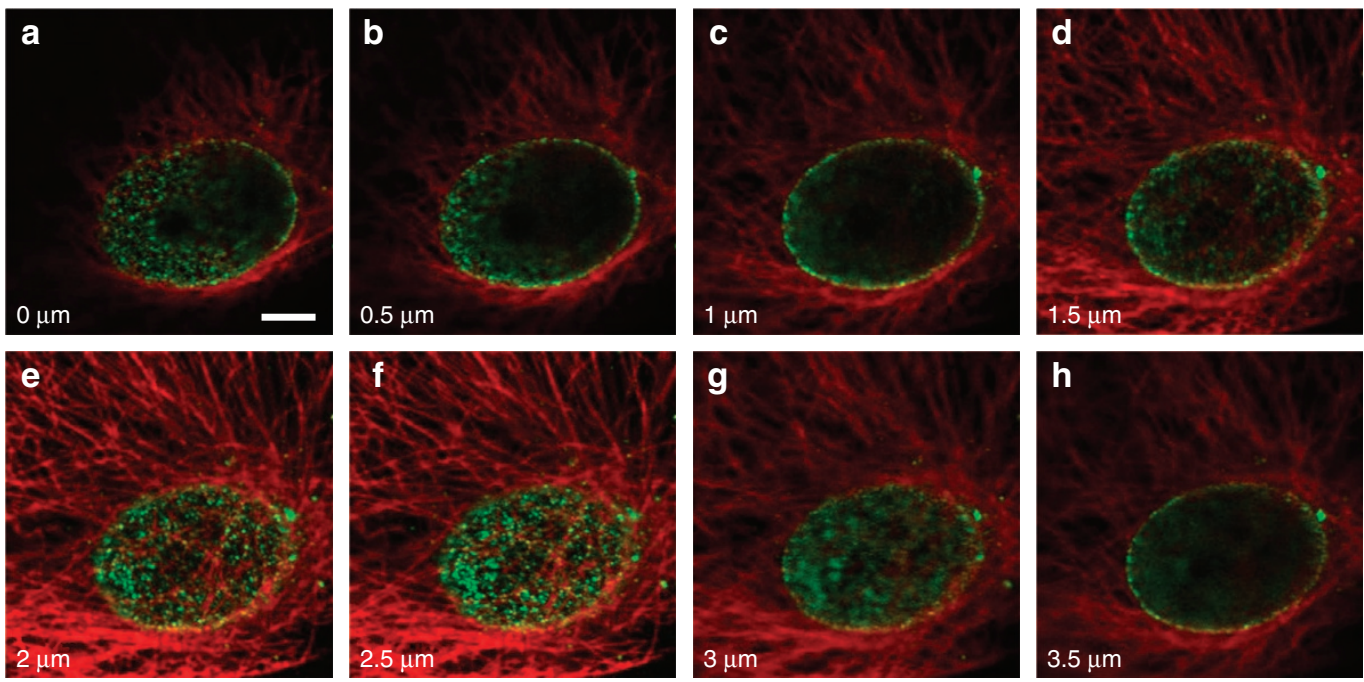

Figure 4 Confocal and MEANS image of the dual-stained Vero cell. The microtubules of the cell are stained with Dylight 650 (pseudo-colored red), and the nuclear pore complex of the cell is stained with Alexa 488 (pseudo-colored green). (a-h) Image series taken from a confocal microscope (Olympus FV1200) with axial step of $0.5 \mu \mathrm{m}$. As observed, MEANS forms at $2.5 \mu \mathrm{m}$ depth in $\mathbf{f}$, in which the nuclear pore proteins are shown clearly as grains. Scale bar $=5 \mu \mathrm{m}$.
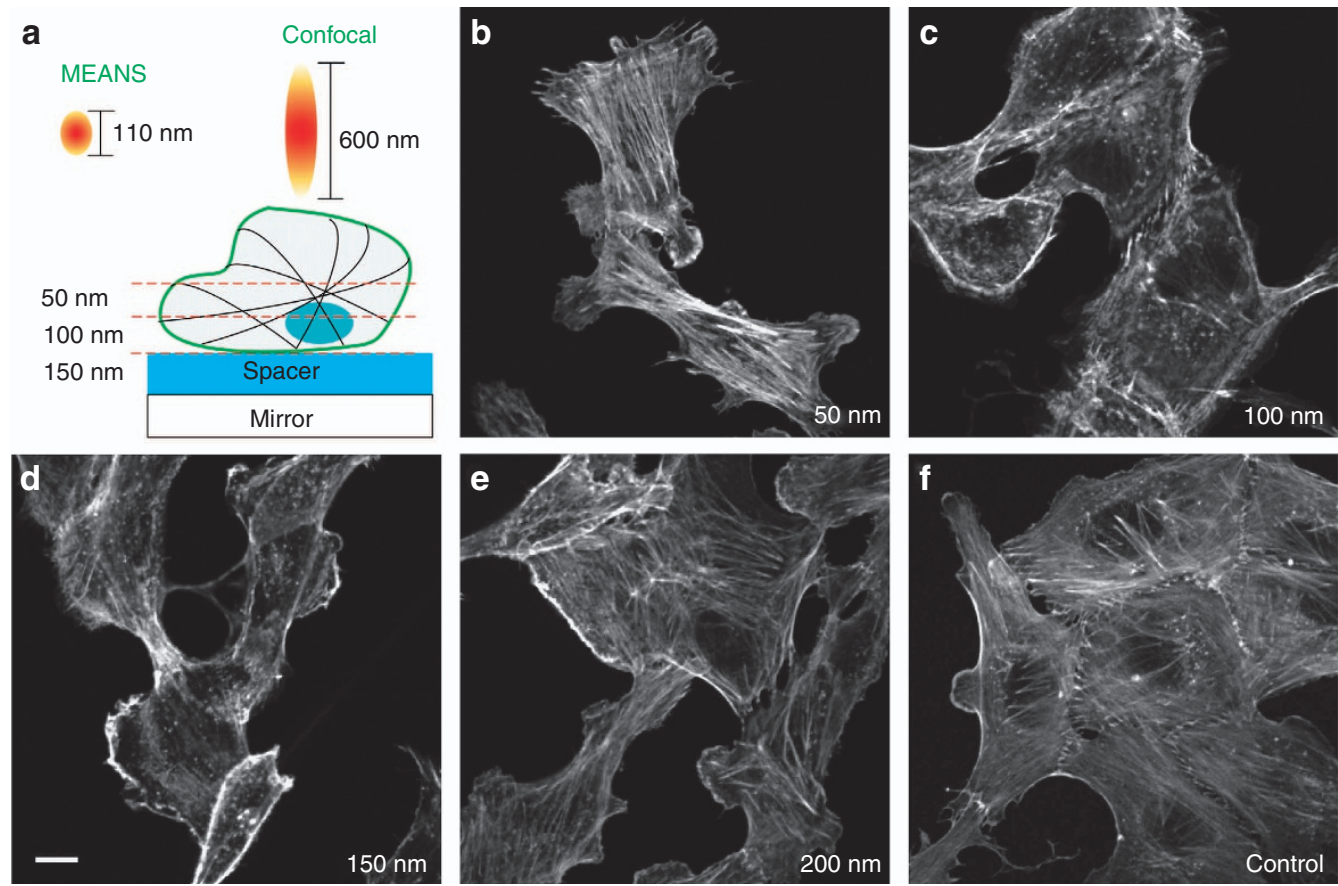

Figure 5 Imaging of the actin filaments with different thickness of silica spacer. Adjusting the spacer thickness can obtain the cross-sectional imaging of different layers of the cell specimen. For MEANS constructive interference, the maximum spacer thickness is 100-150 nm. The images are all of the same size. Scale bar $=10 \mu \mathrm{m}$.

These two model systems illustrate the benefits of the MEANS implementation. The NPC structure (a ring laying parallel to the mirror surface) benefits from increased lateral resolution. According to the Nyquist-Shannon sampling theorem, to resolve a ring structure of $\sim 50 \mathrm{~nm}$ in diameter, a $25-\mathrm{nm}$ imaging resolution is needed. For the hRSV filament (a cylinder lying parallel to the mirror surface), a very thin optical section (less than the diameter of the filament) is required to avoid exciting the entire surface of the filament envelope and to resolve two opposing sides. A more detailed explanation of the geometry of the viral filament can be found in Supplementary Fig. S9.
Because the axial light confinement occurs away from the metal surface, the MEANS method is immune to metal surface quenching ${ }^{13}$. The working distance for MEANS super-resolution can therefore be adjusted by customizing the thickness of the silica coating, which is a standard procedure for commercial protective mirrors. Growing the cell on a coated layer of silica rather than directly on the metal surface provides a more compatible environment for cell growth ${ }^{13}$. Further, with excitation wavelength tuning, MEANS can be extended to axial sectioning of the specimen through MEANS-ESON.

MEANS is compatible with a variety of confocal-based technologies, such as laser scanning, spinning disk and two-photon. In addition, 

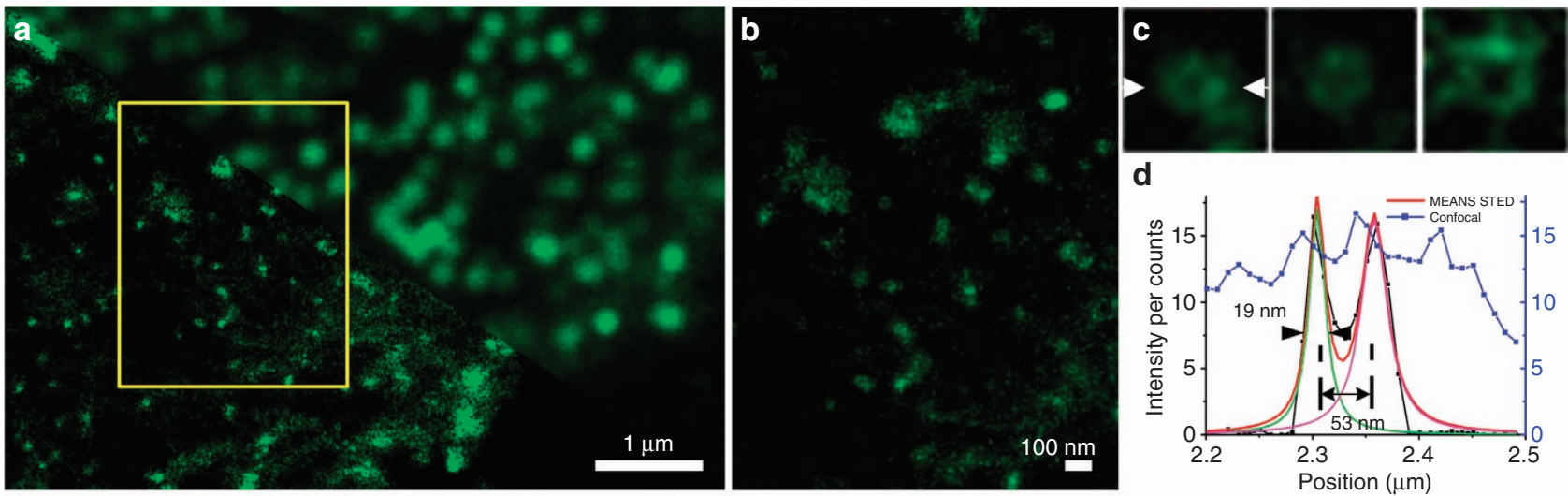

d
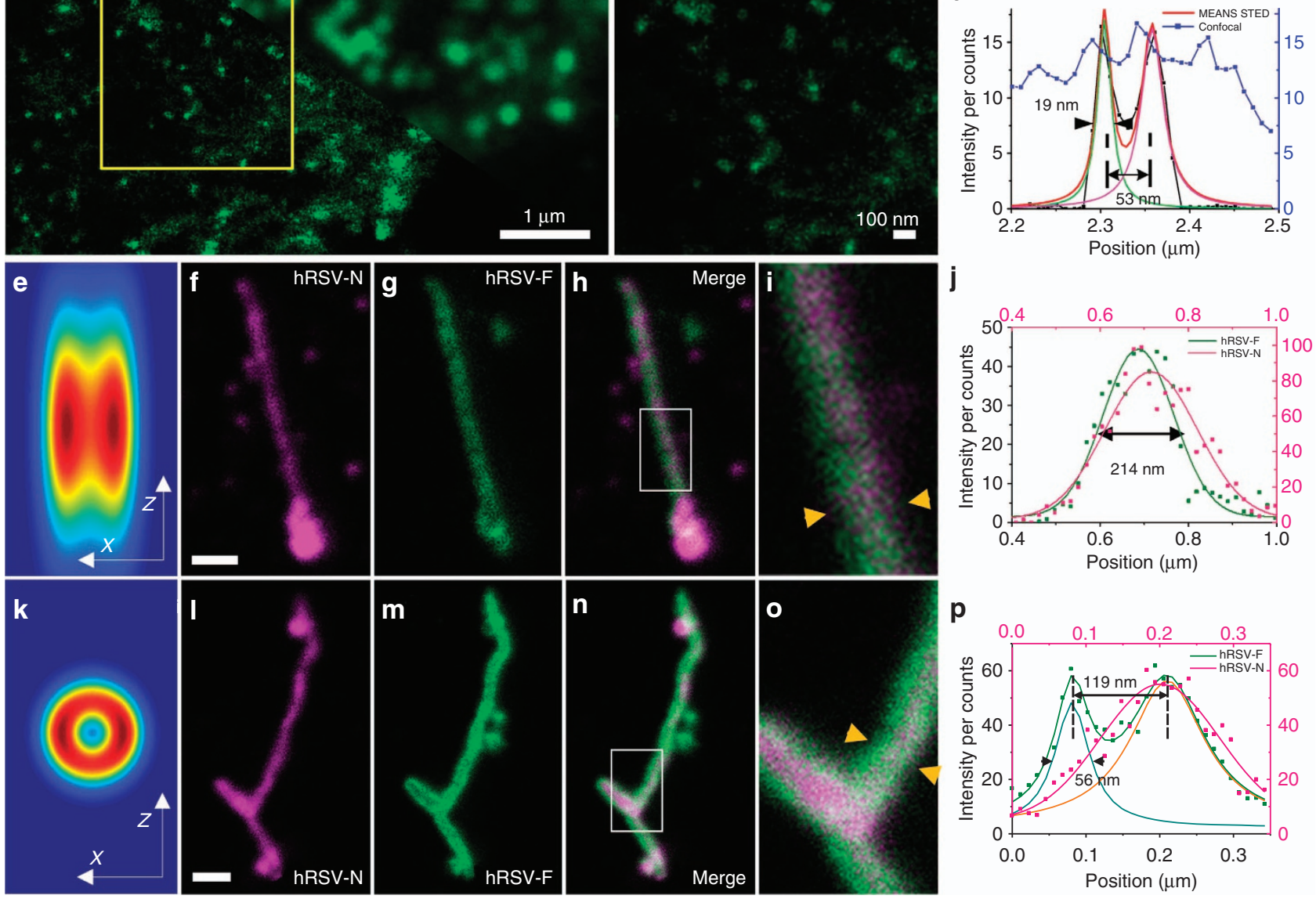

Figure 6 The NPC of a Vero cell (a-c) imaging using confocal (a: upper right) and MEANS-STED (a: lower bottom) modalities, and the hRSV viral filaments imaging via conventional STED ( $\mathbf{f - i}$ ) and MEANS-STED (I-o) modalities. MEANS-STED gives clear lateral resolution enhancement in a. (b) Magnification of the boxed area in $\mathbf{a}$, in which the porous structure of the NPCs can be observed. (c) Magnified individual NPCs. (d) Plot of the intensity distribution along arrows in c. To demonstrate the advantage of the axial confinement of MEANS-STED, the hRSV filaments are imaged with conventional STED (on coverglass, $\mathbf{f}-\mathbf{i}$ ) and MEANS-STED (on mirror, $\mathbf{1 - 0}$ ). The simulation of the convolution of conventional STED PSF vs MEANS-STED PSF with the filament structure is shown in (e) and (k), respectively. The central hollow structure is hard to visualize due to the uniform intensity distribution in e, but in $\mathbf{k}$ it can be observed. $(\mathbf{i}, \mathbf{0})$ Magnifications of the white box areas in $\mathbf{h}$ and $\mathbf{n}$, respectively. The hollow structure of the hRSV-F can be visualized, taking advantage of the optical sectioning of MEANS-STED. (j, p) Intensity plots of the line indicated by the yellow arrows in $\mathbf{i}$ and $\mathbf{o}$, respectively. Gaussian fitting of the data in $\mathbf{j}$ only shows one slope with width of $214 \mathrm{~nm}$, whereas the fitting in $\mathbf{p}$ indicates that the width of the hRSV-F is $\sim 56 \mathrm{~nm}$, and the distance between the split peaks is $~ 119 \mathrm{~nm}$. Scale bar $=500 \mathrm{~nm}(\mathbf{f}, \mathbf{l})$.

MEANS-STED is broadly applicable to PSF engineering-based super-resolution techniques, including the pulsed or time-gated $\mathrm{STED}^{20}, \mathrm{RESOLFT}^{4}$, ground-state depletion, excitation-state absorption, saturation ${ }^{38}$, optical data storage ${ }^{39}$, upconversion ${ }^{40}$ and charge-state depletion ${ }^{41}$. Compared with the other imaging techniques based on the recollection of the signal through interference to confine the axial resolution, including $4 \mathrm{Pi}^{15}, \mathrm{I}^{5} \mathrm{M}^{14}$ and wide-field standingwave microscopy ${ }^{7}$ or mirrors combined with spatial light modulator $^{10,42}$, MEANS is much simpler and does not require precise alignment of the mirror. In addition, MEANS is compatible with conventional sample preparation procedures, which is advantageous over virtual imaging 43 .

\section{CONCLUSION}

It has been demonstrated that with axial confinement, such as TIRF, improved image contrast can be obtained. We found that through the straightforward use of a mirror beneath the specimen, we can generate an interference-enhanced layer away from the mirror reflective surface with a thickness of $\sim 110 \mathrm{~nm}$. A 3.6-fold local EM field enhancement can be obtained, resulting in an increased SBR. Although MEANS is very similar to the $4 \mathrm{Pi}$ approach, when using MEANS, the height of the interference-enhanced layer is constant during mirror translation of $0.4 \mu \mathrm{m}$, which largely relieves the requirement of ultra-precise system alignment. Furthermore, MEANS is compatible with a series of confocal modalities, such as two-photon, spinning disk and laser scanning. When MEANS is applied to STED, both the lateral and axial resolution can be improved simultaneously. The ring structure of the inner NPC structure (diameter of $53 \mathrm{~nm}$ ) can be visualized with a resolution of $\sim 19 \mathrm{~nm}$. To the best of our knowledge, this resolution is among the best recorded for STED super-resolution in biological specimens. Taking advantage of the thin axial depth of MEANS, we can discern the tubular structure of the hRSV-F protein with a diameter of 
$\sim 120 \mathrm{~nm}$. The capability of achieving optical sectioning and high lateral resolution is particularly appealing to biological applications because the specimens are usually prone to strong light power.

\section{CONFLICT OF INTEREST}

The authors declare no conflict of interest.

\section{AUTHOR CONTRIBUTIONS}

HX and PX conceived the project and conducted the simulation, PX, DJ and PJS designed the experiments and managed the project, EA and PJS prepared the biological cell specimens, XY and PX performed the imaging experiments and data analysis. DJ, XY, HX, PJS and PX wrote the manuscript. All authors participated in discussion and editing of the manuscript.

\section{ACKNOWLEDGEMENTS}

The fluorescent nanodiamond sample was a gift from Huan-cheng Chang (Institute of Atomic and Molecular Sciences, Academia Sinica). We thank Jihong Liu from Leica (China) and Chunyan Shan (Peking University) for their help with the Leica TCS SP8 STED 3X, Weijia Yang (Wuhan YSL Photonics) for helpful discussion about the white light laser, and Xu Zhang (Tsinghua University) for helpful discussion about sample preparation. This research is supported by the National Instrument Development Special Program (2013YQ03065102), the '973' Major State Basic Research Development Program of China (2011CB809101), the Natural Science Foundation of China $(31327901,61475010,61428501)$ and the Australian Research Council Centre of Excellence for Nanoscale BioPhotonics (CE140100003). Support for E Alonas was provided by the National Institute of Health (GM094198 to PJS).

1 Betzig E, Patterson GH, Sougrat R, Lindwasser OW, Olenych S et al. Imaging intracellular fluorescent proteins at nanometer resolution. Science 2006; 313: 1642-1645.

2 Axelrod D. Total internal reflection fluorescence microscopy in cell biology. Traffic 2001; 2: 764-774.

3 Orrit M. Nobel prize in chemistry: celebrating optical nanoscopy. Nat Photonics 2014; 8: 887-888.

4 Hell SW. Far-field optical nanoscopy. Science 2007; 316: 1153-1158.

5 Hao X, Kuang CF, Gu ZT, Wang YF, Li S et al. From microscopy to nanoscopy via visible light. Light Sci App/ 2013; 2: e108, doi:10.1038/lsa.2013.64.

6 Schmidt R, Wurm CA, Jakobs S, Engelhardt J, Egner A et al. Spherical nanosized focal spot unravels the interior of cells. Nat Methods 2008; 5: 539-544.

7 Bailey B, Farkas DL, Taylor DL, Lanni F. Enhancement of axial resolution in fluorescence microscopy by standing-wave excitation. Nature 1993; 366: 44-48.

8 Swan AK, Moiseev LA, Cantor CR, Davis B, Ippolito SB et al. Toward nanometer-scale resolution in fluorescence microscopy using spectral self-interference. IEEE J Select Top Quant Electron 2003; 9: 294-300.

9 Amor R, Mahajan S, Amos WB, McConnell G. Standing-wave-excited multiplanar fluorescence in a laser scanning microscope reveals 3D information on red blood cells. Sci Rep 2014; 4: 7359.

10 Mudry E, Le Moal E, Ferrand P, Chaumet PC, Sentenac A. Isotropic diffraction-limited focusing using a single objective lens. Phys Rev Lett 2010; 105: 203903.

11 Deguchi T, Koho S, Näreoja T, Hänninen P. Axial super-resolution by mirror-reflected stimulated emission depletion microscopy. Opt Rev 2014; 21: 389-394.

12 Elsayad K, Urich A, Tan PS, Nemethova M, Small JV et al. Spectrally coded optical nanosectioning (SpecON) with biocompatible metal-dielectric-coated substrates. Proc Natl Acad Sci USA 2013; 110: 20069-20074.

13 Chizhik AI, Rother J, Gregor I, Janshoff A, Enderlein J. Metal-induced energy transfer for live cell nanoscopy. Nat Photonics 2014; 8: 124-127.

14 Gustafsson M, Agard D, Sedat J. I ${ }^{5}$ M: 3D widefield light microscopy with better than $100 \mathrm{~nm}$ axial resolution. J Microsc 1999; 195: 10-16.

15 Hell SW, Lindek S, Cremer C, Stelzer EHK. Measurement of the 4Pi-confocal point spread function proves $75 \mathrm{~nm}$ axial resolution. Appl Phys Lett 1994; 64: 1335-1337.

16 Li S, Kuang CF, Hao X, Gu ZT, Liu X. Generation of a 3D isotropic hollow focal spot for single-objective stimulated emission depletion microscopy. J Opt 2012; 14: 085704.
17 You ST, Kuang CF, Rong ZH, Liu X, Ding ZH. Isotropic superresolution imaging for fluorescence emission difference microscopy. Appl Opt 2014; 53: 7838-7844.

18 Zhao ZW, Roy R, Gebhardt JCM, Suter DM, Chapman AR et al. Spatial organization of RNA polymerase II inside a mammalian cell nucleus revealed by reflected light-sheet superresolution microscopy. Proc Natl Acad Sci USA 2014; 111: 681-686.

19 Wurm CA, Neumann D, Schmidt R, Egner A, Jakobs S. Sample preparation for STED microscopy. Methods Mol Biol 2010; 591: 185-199.

20 Vicidomini G, Moneron G, Han KY, Westphal V, Ta H et al. Sharper low-power STED nanoscopy by time gating. Nat Methods 2011; 8: 571-573.

21 Tokunaga M, Imamoto N, Sakata-Sogawa K. Highly inclined thin illumination enables clear single-molecule imaging in cells. Nat Methods 2008; 5: 159-161.

22 Bewersdorf J, Schmidt R, Hell SW. Comparison of $\mathrm{I}^{5} \mathrm{M}$ and 4Pi-microscopy. J Microsc 2006; 222: 105-117.

23 Wilson T, Sheppard C. Theory and Practice of Scanning Optical Microscopy. London: Academic Press; 1984.

24 Watanabe T, Igasaki Y, Fukuchi N, Sakai M, Ishiuchi S et al. Formation of a doughnut laser beam for super-resolving microscopy using a phase spatial light modulator. Opt Eng 2004; 43: 1136-1143.

25 Gu M. Advanced Optical Imaging Theory, vol. 75. Berlin: Springer Science \& Business Media; 2000.

26 Xie H, Liu YJ, Santangelo PJ, Jin DY, Xi P. Analytical description of high-aperture STED resolution with 0-2 $\pi$ vortex phase modulation. J Opt Soc Am A 2013; 30: 1640-1645.

27 Westphal V, Hell SW. Nanoscale resolution in the focal plane of an optical microscope. Phys Rev Lett 2005; 94: 143903.

28 Alber F, Dokudovskaya S, Veenhoff LM, Zhang WZ, Kipper J et al. The molecular architecture of the nuclear pore complex. Nature 2007; 450: 695-701.

29 Guan T, Müller S, Klier G, Panté N, Blevitt JM et al. Structural analysis of the p62 complex, an assembly of $\mathrm{O}$-linked glycoproteins that localizes near the central gated channel of the nuclear pore complex. Mol Biol Cell 1995; 6: 1591-1603.

30 Grossman E, Medalia O, Zwerger M. Functional architecture of the nuclear pore complex. Annu Rev Biophys 2012; 41: 557-584.

31 Szymborska A, de Marco A, Daigle N, Cordes VC, Briggs JAG et al. Nuclear pore scaffold structure analyzed by super-resolution microscopy and particle averaging. Science 2013; 341: 655-658.

32 Donnert G, Keller J, Medda R, Andrei MA, Rizzoli So et al. Macromolecular-scale resolution in biological fluorescence microscopy. Proc Natl Acad Sci USA 2006; 103 : 11440-11445.

33 Wildanger D, Medda R, Kastrup L, Hell SW. A compact STED microscope providing 3D nanoscale resolution. J Microsc 2009; 236: 35-43.

34 Göttfert F, Wurm CA, Mueller V, Berning S, Cordes VC et al. Coaligned dual-channel STED nanoscopy and molecular diffusion analysis at $20 \mathrm{~nm}$ resolution. Biophys J 2013; 105: LO1-LO3.

35 Kolmakov K, Wurm CA, Meineke DNH, Göttfert F, Boyarskiy VP et al. Polar red-emitting rhodamine dyes with reactive groups: synthesis, photophysical properties, and two-color STED nanoscopy applications. Chemistry 2014; 20: 146-157.

36 Wurm CA, Kolmakov K, Göttfert F, Ta H, Bossi M et al. Novel red fluorophores with superior performance in STED microscopy. Opt Nanoscopy 2012; 1: 1-7.

37 Alonas E, Lifland AW, Gudheti M, Vanover D, Jung J et al. Combining single RNA sensitive probes with subdiffraction-limited and live-cell imaging enables the characterization of virus dynamics in cells. ACS Nano 2014; 8: 302-315.

38 Fujita K, Kobayashi M, Kawano S, Yamanaka M, Kawata S. High-resolution confocal microscopy by saturated excitation of fluorescence. Phys Rev Lett 2007; 99: 228105

39 Gan ZS, Cao YY, Evans RA, Gu M. Three-dimensional deep sub-diffraction optical beam lithography with $9 \mathrm{~nm}$ feature size. Nat Commun 2013; 4: 2061.

40 Kolesov R, Reuter R, Xia KW, Stöhr R, Zappe A et al. Super-resolution upconversion microscopy of praseodymium-doped yttrium aluminum garnet nanoparticles. Phys Rev B 2011; 84: 153413.

41 Chen XD, Zou CL, Gong ZJ, Dong CH, Guo GC et al. Subdiffraction optical manipulation of the charge state of nitrogen vacancy center in diamond. Light Sci Appl 2015; 4: e230, doi:10.1038/lsa.2015.3.

42 Schnitzbauer J, McGorty R, Huang B. 4Pi fluorescence detection and 3D particle localization with a single objective. Opt Express 2013; 21: 19701-19708.

43 Wang ZB, Guo W, Li L, Luk'yanchuk B, Khan A et al. Optical virtual imaging at $50 \mathrm{~nm}$ lateral resolution with a white-light nanoscope. Nat Commun 2011; 2: 218.

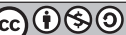

This work is licensed under a Creative Commons AttributionNonCommercial-ShareAlike 4.0 International License. The images or other third party material in this article are included in the article's Creative Commons license, unless indicated otherwise in the credit line; if the material is not included under the Creative Commons license, users will need to obtain permission from the license holder to reproduce the material. To view a copy of this license, visit http:// creativecommons.org/licenses/by-nc-sa/4.0/

Supplementary Information for this article can be found on the Light: Science \& Applications' website (http://www.nature.com/lsa). 